\title{
PHYCOERYTHROCYANINS FROM Westiellopsis prolifica AND Nostoc rivulare: CHARACTERIZATION OF THE PHYCOVIOLOBILIN CHROMOPHORE IN BOTH STATES*
}

\author{
P. S. Maruthi SaI ${ }^{1}$, S. Siebzehnrübl ${ }^{2}$, S. Mahajan ${ }^{1}$ and H. Scheer ${ }^{2} \dagger$ \\ ${ }^{1}$ School of Life Sciences, Jawaharlal Nehru University, New Delhi 110067, India and ${ }^{2}$ Botanisches \\ Institut der Universität, Menzinger Str. 67, 8000 München 19, Fed. Rep. Germany
}

(Received 14 September 1990; accepted 5 June 1991)

\begin{abstract}
Phycoerythrocyanin or fractions enriched in it have been isolated from the filamentous cyanobacteria, Westiellopsis prolifica ARM 365 and Nostoc rivulare ARM 212. Both show the photoreversible photochromism (difference maxima at 503 and $570 \mathrm{~nm}$ ) characteristic of this pigment, which is related to the phycoviolobilin chromophore on the $\alpha$-subunit. Native phycoerythrocyanin and its $\beta$ subunit show little if any reversible photochemistry in the $600-620 \mathrm{~nm}$ region, where the phycocyanobilin chromophores absorb maximally. Instead the phycocyanobilin chromophores are bleached irreversibly. At the same time, the data show that reversible photochemistry is a useful analytical tool to detect phycoerythrocyanin in cyanobacterial extracts. Fluorescence measurements indicate that: (i) the $510 \mathrm{~nm}$ absorbing isomer of the violobilin chromophore has only little fluorescence; and (ii) the energy transfer from the violobilin chromophores to the cyanin chromophores is efficient only in the $570 \mathrm{~nm}$ form.
\end{abstract}

\section{INTRODUCTION}

Cyanobacteria are the oxygen evolving photosynthetic prokaryotes possessing brilliantly coloured phycobiliproteins involved in light harvesting and excitation energy transfer to the reaction centres (Scheer, 1986). Phycoerythrocyanin (PEC)市, one such biliprotein, also shows photoreversible photochemistry reminiscent of the plant photoreceptor, phytochrome, but with an orange/green rather than red/far-red action spectrum (Björn, 1979; Kufer and Björn, 1989; Siebzehnrübl et al., 1989). It has been found in several filamentous cyanobacteria (Bryant et al., 1976; MacColl and Guard-Friar, 1987; Bryant, 1982). It is structurally related to C-phycocyanin (C-PC) (Zuber, 1986; Dürring et al., 1990), but the $\alpha$-subunit carries an unusual phycoviolobilin§ instead of the phycocyanobilin chromophores which are present on the $\beta$-subunit only. Phycoerythrocyanin is already photoreactive in its native state (Kufer and Björn, 1989). Björn (1979) first suggested a relationship between phycochrome $b$, so far identified only in PEC containing blue-green algae, and the $\alpha$-subunit of PEC. Siebzehnrübl et al. (1989) discussed in detail the photochemistry of PEC from Mastigocladus (M.) laminosus in various

* Dedicated to Professor W. Wehrmeyer on the occasion of his 60 th birthday.

tTo whom correspondence should be addressed.

$\ddagger$ Abbreviations: APC, allophycocyanin; PBSomes, phycobilisomes; PC, phycocyanin; PEC, phycoerythrocyanin; PMT, photomultiplier tube.

\$This chromophore nomenclature follows the one used for other biliprotein chromophores. In the literature, the PEC chromophore on the $\alpha$-subunit has also been termed as phycobiliviolin, PXB (Bishop et al., 1987). aggregation states.

In continuing a systematic study on the photoreactivity of PEC, we have now further characterized PEC from two other species, e.g. the filamentous and heterocystous cyanobacteria Westiellopsis prolifica ARM 365 and Nostoc rivulare ARM 212. Here we report the behaviour of PEC observed during photochemical changes in various states ranging from PEC in intact phycobilisomes to the isolated native forms. Further, we also present the fluorescence properties of the phycoviolobilin chromophore on the $\alpha$-subunit of PEC in its two forms.

\section{MATERIALS AND METHODS}

Nostoc rivulare ARM 212 and Westiellopsis prolifica ARM 365 were obtained as agar slants from the Nationa Facility for Blue-Green Algae, Indian Agricultural Research Institute, New Delhi, India. They were grown in $5-10 \mathrm{~L}$ batch cultures at $28^{\circ} \mathrm{C}$ under irradiation with fluorescent lamps $(\approx 3000 \mathrm{~lx}$ ) in BG-11 medium (Stanier et al., 1971). After 10-12 days, the cells were harvested by vacuum filtration and washed thoroughly in potassium phosphate buffer (1 M, pH 7.0). Phycobilisomes (PBSomes) were isolated according to Gantt et al. (1979) or by the following modification of the procedure of Menon et al. (1988) which yields intact PBSomes from these species without mechanical breakage. Washed cells (1-1.2 $\mathrm{g}$ wet weight $10 \mathrm{~mL}$ ) were suspended in potassium phosphate buffer ( $1 M, \mathrm{pH} 7.0$ ) containing PMSF ( $1 \mathrm{mM})$ and homogenized with a Potter homogenizer. Neat Triton$\mathrm{X} 100$ was added to $2 \%$, or alternatively CTAB to $0.01 \%$. The cell suspensions were vigorously stirred on a magnetic stirrer for about $4 \mathrm{~h}$ at room temperature, and then centrifuged $\left(35000 \mathrm{~g}\right.$, at $\left.20^{\circ} \mathrm{C}\right)$. The supernatants were carefully removed with Pasteur pipettes, and dialysed overnight at room temperature against the $1 M$ buffer. The preparations were centrifuged again $\left(35000 \mathrm{~g}\right.$, at $\left.20^{\circ} \mathrm{C}\right)$ to remove scattering particles, and checked for intactness of PBSomes by fluorescence spectroscopy. All the remaining 


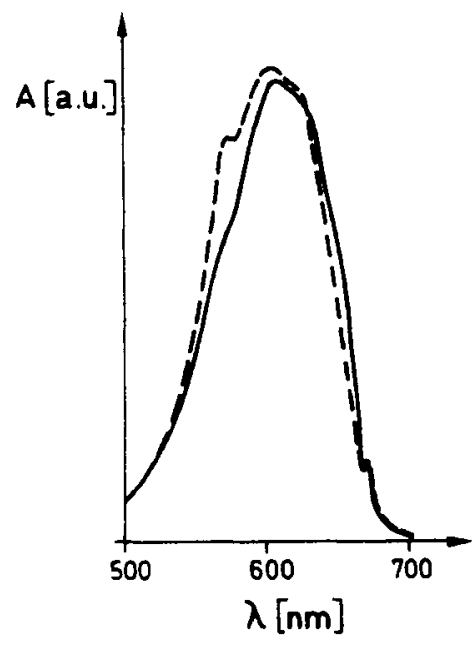

Figure 1. Absorption spectra of intact PBSomes in potassium phosphate buffer $(1 \mathrm{M}, \mathrm{pH} 7.0)$ of $W$. prolifica (---) and $N$. rivulare (-).

steps were performed according to Gantt et al. (1979).

For obtaining PEC, the intact PBSomes were dissociated by dialysing overnight against potassium phosphate buffer $(5 \mathrm{mM}, \mathrm{pH} 7.0)$ at $4^{\circ} \mathrm{C}$. The dissociated PBSomes were applied onto DEAE cellulose (columns DE 52, Whatman) and constituent biliproteins were eluted with a step gradient (10-200 $\mathrm{m} M$ potassium phosphate buffer, $\mathrm{pH} 7.0$ ) Alternatively, dissociated PBSomes were subjected to sucrose density ultracentrifugation $(7-17 \%$ linear gradient, $48 \mathrm{~h}, 4^{\circ} \mathrm{C}, 238000 \mathrm{~g}$ ) and were separated into differen fractions of biliproteins. All fractions indicating the presence of PEC (absorption band at $\approx 575 \mathrm{~nm}$ ) were analysed for light induced absorbance changes (see below) and by SDS-PAGE (Laemmli, 1970) and pooled accordingly. Monomers of PEC were obtained by addition of KSCN to $1 M$ (MacColl et al., 1981) to the PEC trimers.

Absorption spectra were obtained at room temperature unless specified otherwise. Steady state absorption spectra were recorded on either Shimadzu UV 260, Beckman DU 70 , Hewlett-Packard 8451A diode array, or Shimadzu UV 3000 spectrophotometers fitted with thermostats. Absorption difference spectra were recorded on a SigmaZWS 11 dual wavelength spectrophotometer in split-beam mode with the cuvettes thermostatted at $15^{\circ} \mathrm{C}$. This spectrophotometer, with the PMT mounted immediately behind the cuvette, was also used to measure the absorption of scattering samples. The steady state fiuorescence excitation and emission spectra were recorded on Shimadzu RF 540, Perkin-Eimer MPF4 or Spex Fluorolog 221 spectrofluorometers in $90^{\circ}$ geometry. Maximum absorption was $<0.1 \mathrm{~cm}^{-1}$ (square cells $1 \times 1 \mathrm{~cm}$ ). All spectra are corrected for instrument response.

The photochemistry was tested by difference spectroscopy. After a saturating pre-irradiation (10 $\mathrm{min}$, $500 \mathrm{~nm}$ light), the samples were irradiated for $8 \mathrm{~min}$ with $600 \mathrm{~nm}$ light. Reversibility was checked by post-irradiation for $10 \mathrm{~min}$ with $500 \mathrm{~nm}$ light. Quantitative data are given as $\triangle \Delta A$ values $(\%)$. Details of the assay are given by Siebzehnrübl et al. (1989).

\section{RESULTS}

Whole cells of both species of cyanobacteria show prominent absorptions around $675 \mathrm{~nm}$ (chlorophyl a) and $620 \mathrm{~nm}$ (PC) (not shown). A distinct shoulder around $580 \mathrm{~nm}$ in $W$. prolifica indicates

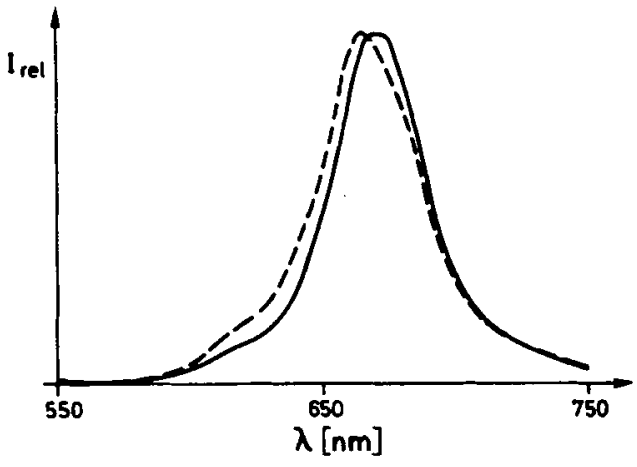

Figure 2. Fluorescence emission spectra of intact PBSomes from $W$. prolifica (---) and $N$. rivulare $(\longrightarrow)$. Excitation at $540 \mathrm{~nm}$, slit widths $5 \mathrm{~nm}$, normalized to the same maximum emission.

the presence of PEC. In $N$. rivulare, the 620 absorption is broadened, but shows no distinct shoulder, which agrees with the low level of PEC (see below).

The absorption spectrum of intact PBSomes from W. prolifica (Fig. 1) shows a maximum around $608 \mathrm{~nm}$ with a prominent red shoulder, in addition to the one at the short-wavelength side. The spectrum of the $N$. rivulare PBSomes is similar, but here the $575 \mathrm{~nm}$ shoulder is barely visible (Fig. 1). The fluorescence emission spectra of intact PBSomes (Fig. 2) were almost identical from both species with emission around $670-680 \mathrm{~nm}$. This indicates that the PBSomes are energetically well-coupled (Gantt et al., 1979). The intact PBSomes of W. prolifica were dissociated overnight and the extent of dissociation was checked by fluorescence emission spectroscopy (Fig. 3). Emissions in dissociated PBSomes at 580 and $645 \mathrm{~nm}$, when excited at $545 \mathrm{~nm}$, reveal the detachment of PEC, PC and APC with a concomitant loss of energy transfer from PEC to PC to APC. Note that the PEC fluorescence maximum is close to the wavelength of the absorption maximum of PEC in intact PBSomes.

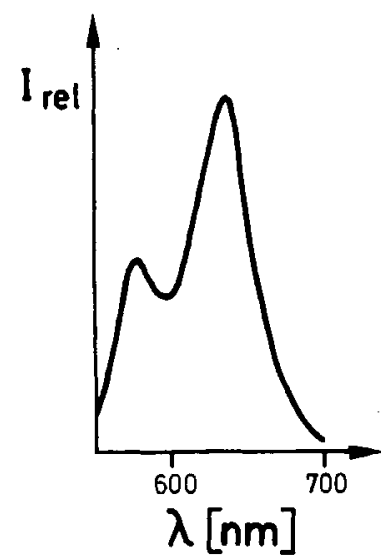

Figure 3. Fluorescence emission spectrum of dissociated PBSomes from $W$. prolifica in potassium phosphate buffer ( $5 \mathrm{mM}, \mathrm{pH} 7.0$ ). Excitation wavelength $540 \mathrm{~nm}$. 
Table 1. Reversible photochemistry of PEC from $W$. prolifica and $N$. rivulare in different states. Irradiation protocol as in Figs. 6 and 7. $\Delta \Delta A$ denotes the ratio of the amplitude of the s-shaped difference band [see Fig. 6(a)] and the $575 \mathrm{~nm}$ absorption after saturating pre-irradiation with green light

\begin{tabular}{|c|c|c|c|c|c|}
\hline \multirow[b]{2}{*}{ Sample } & \multirow[b]{2}{*}{ Buffer* } & \multicolumn{2}{|c|}{ Difference etrema (nm) } & \multirow{2}{*}{$\frac{\Delta \Delta A \dagger}{(\%)}$} & \multirow[b]{2}{*}{ Reversibility } \\
\hline & & Orange & Green & & \\
\hline \multicolumn{6}{|l|}{ W. prolifica } \\
\hline Intact PBSomes & $1 M$ & - & - & - & - \\
\hline Dissociated PBSomes & $5 \mathrm{mM}$ & 567 & 503 & 6.1 & \\
\hline $\operatorname{PEC}(X)$ & $50 \mathrm{mM}$ & 570 & 508 & 84.8 & Total \\
\hline PEC & $100 \mathrm{mM}$ & 570 & 508 & 17 & Total \\
\hline PEC & $100 \mathrm{~m} M / \mathrm{KSCN} 1 M$ & 567 & 505 & 41 & Total \\
\hline \multicolumn{6}{|l|}{ N. rivulare } \\
\hline Intact PBSomes & $1 M$ & - & - & - & - \\
\hline Dissociated PBSomes & $5 \mathrm{mM}$ & 569 & 505 & 3.3 & Total \\
\hline $\mathrm{PEC} \ddagger$ & $1 M$ & 571 & 506 & 2.5 & - \\
\hline PEC $\ddagger$ & $100 \mathrm{~m} M$ & 569 & 506 & 23.8 & Total \\
\hline
\end{tabular}

* Potassium phosphate buffer, pH 7.

+Absorption difference amplitude, nomalized to $A_{\max }$ of sample, see Siebzehnrübl et al. (1989).

$\ddagger$ Fraction enriched in PEC, see text and Figs. 4 and 6 (c).

During the isolation of PEC from $N$. rivulare PBSomes, a separate band containing PEC was observed in the region of $0.5-1.0 \mathrm{M}$ on the sucrose density step gradients apart from the main PBSome band in the region of $2.0 \mathrm{M}$. The PEC containing band, when checked for the photochemistry indicated $\approx 2.5 \%$ photochemical signal $(\Delta \Delta A$, Table 1 ), but we could observe a photochemistry similar to that of PEC monomers when KSCN was added to $1 \mathrm{M}$. We thus report the presence of PEC from $N$. rivulare.

The dissociated PBSomes were subjected to chromatography on DEAE-cellulose ion exchanger for separation of component phycobiliproteins. The absorption spectra of various fractions of PEC are shown in Fig. 4. The fractions of PEC from $W$. prolifica eluting at $50 \mathrm{~m} M$ phosphate buffer, resemble spectroscopically that of an $\alpha$-subunit of PEC after $600 \mathrm{~nm}$ irradiation (Kufer and Björn, 1989) (Fig. 4). It lacks the characteristic absorption of the two cyanobilin-chromophores on the $\beta$-subunit. The nature of this fraction, which contains additional polypeptides, is presently under investigation. The subsequent fractions eluting at $100 \mathrm{mM}$ phosphate have an absorption like that of PEC trimers from Mastigocladus laminosus (Siebzehnrübl et al., 1989). The absorption spectrum of the $N$. rivulare pigment fraction eluting first from DEAE-cellulose is shown in Fig. 4. It is dominated by PC $\left(\lambda_{\max }=620 \mathrm{~nm}\right)$, but now shows a prominent

* The photochemical response of PEC is consistently higher upon irradiation at 600 than at $580 \mathrm{~nm}$, although the absorption is much higher at $580 \mathrm{~nm}$. It is possible that this is caused by the presence of intermediates in the photoprocess which are in photochemical equilib rium with the $570 \mathrm{~nm}$ form of PEC, or by an "uphill" energy transfer from the lower lying phycocyanobilin chromophores

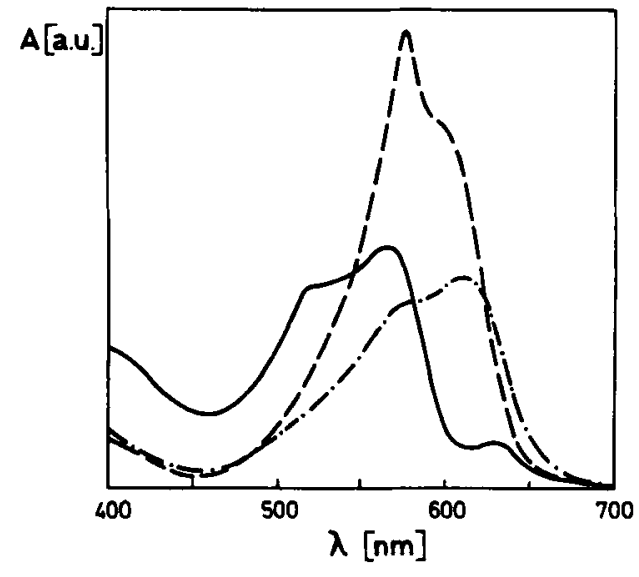

Figure 4. Absorption spectra of PEC fractions obtained on DEAE-cellulose columns. $\mathrm{PEC}(\mathrm{X})$ in potassium phosphate buffer $(50 \mathrm{mM}, \mathrm{pH} 7.0)$ from $W$. prolifica $(\stackrel{\mathrm{L}}{\longrightarrow})$. PEC from $W$. prolifica in potassium phosphate buffer (100 $\mathrm{m} M, \mathrm{pH} 7.0)(--)$. PEC containing fraction from $N$. rivulare in potassium phosphate buffer $(100 \mathrm{~m} M, \mathrm{pH} 7.0)$

$$
(\cdots)
$$

shoulder at $575 \mathrm{~nm}$ indicative of PEC.

The enrichment of PEC was also followed by its photoreversible photochromic response to orange $(600 \mathrm{~nm})^{*}$ and green light $(500 \mathrm{~nm})$. Photochemical changes in dissociated PBSomes and all the fractions of PEC from $W$. prolifica and $N$. rivulare in which in the presence of PEC was indicated by absorption are summarized in Table 1 . The most intense absorption change was observed with $\operatorname{PEC}(\mathrm{X})$ (Fig. $5)$ from $W$. prolifica. The amplitude of the difference signals [Fig. 6(a)] is 5 times that of the trimeric PEC from the same organism. However, the shape, exact positions of the maxima, and relative areas of the positive and negative difference bands are somewhat different in the main PEC fraction from 


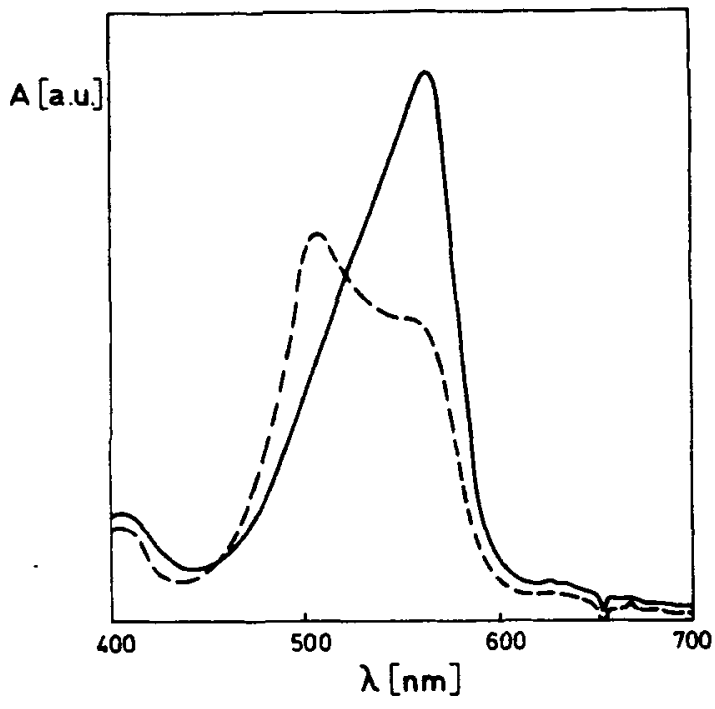

Figure 5. Absorption spectra of native $\operatorname{PEC}(X)$ from $W$ prolifica after saturating pre-irradiation with $500 \mathrm{~nm}$ (-) and saturating irradiation with $600 \mathrm{~nm}$ light $(\cdots-)$ $(8 \min$ each).

this organism [Fig. 6(b)]. Apart from these variations, the difference spectra are similar to those observed in PEC from $M$. laminosus (Siebzehnrübl et al., 1989; Kufer and Björn, 1989). The difference signal of the enriched fraction from $N$. rivulare clearly shows that this organism contains PEC too, albeit in much smaller amounts [Fig. 6(c)].

The intense absorption changes observed in $\mathrm{PEC}(\mathrm{X})$ from $W$. prolifica prompted us to study the contribution of the $\alpha$-subunit of PEC in its two forms to the fluorescence. For this, the $510 \mathrm{~nm}$ absorbing form was first enriched by orange preirradiation. Fluorescence emission spectra were then recorded for excitation at 540 and $480 \mathrm{~nm}$. The band shape and position of the emission is the same when this sample is excited at 480 and $540 \mathrm{~nm}$ (Fig. 7). However, although the absorption at $480 \mathrm{~nm}$ is as intense as that at $540 \mathrm{~nm}$ (see Fig. 5), the emission is greatly diminished upon $480 \mathrm{~nm}$ excitation. These fluorescence yields parallel the absorption of the $565 \mathrm{~nm}$ absorbing form of the phycoviolobilin chromophore, which is much larger at $540 \mathrm{~nm}$ than at $480 \mathrm{~nm}$. By contrast, the $510 \mathrm{~nm}$ absorbing form of the chromophore has much higher absorption at $480 \mathrm{~nm}$ than at $540 \mathrm{~nm}$ (Scheer, unpublished). This indicates that the $565 \mathrm{~nm}$ absorbing form of phycoviolobilin chromophore contributes predominantly to fluorescence, whereas the $510 \mathrm{~nm}$ form is "silent".

\section{DISCUSSION}

Action spectra have been obtained for a variety of light-controlled responses including chromatic adaptation and photomorphogenesis, and the putative photoreceptors have accordingly been called
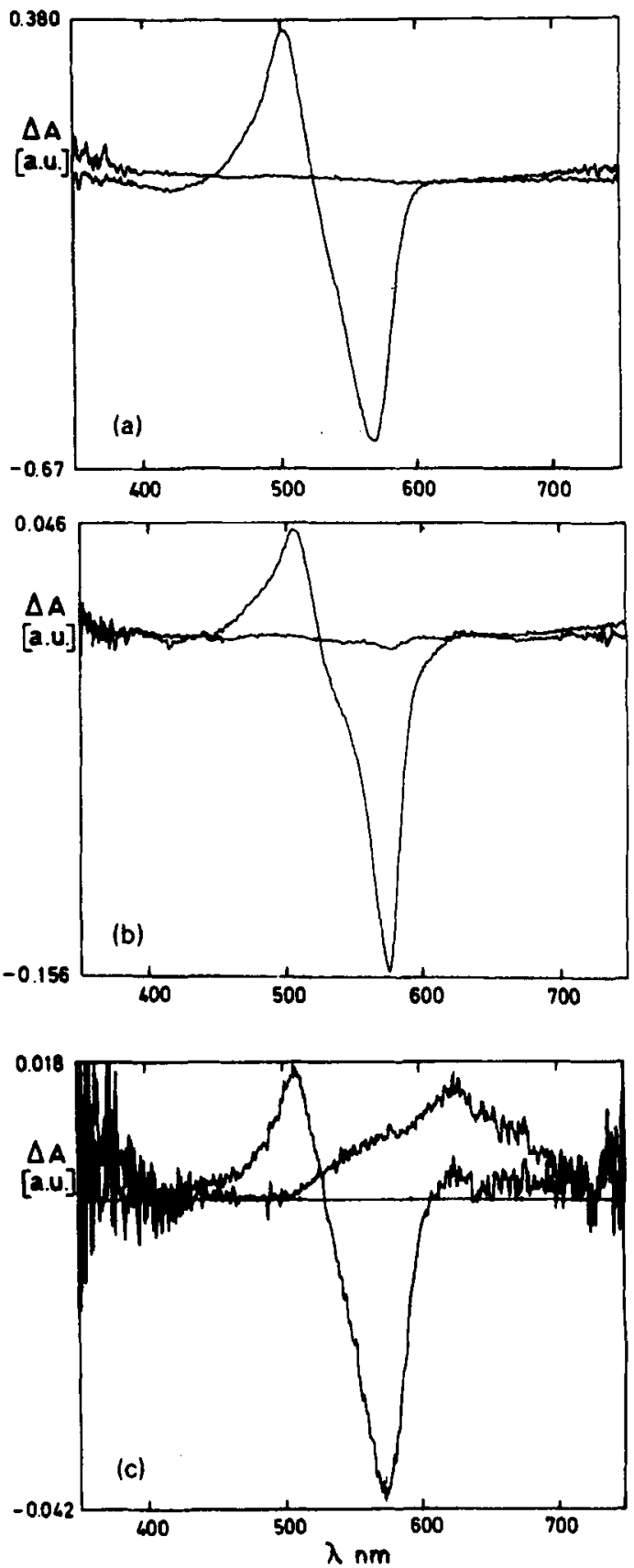

Figure 6. Absorption difference spectra (orange-irradiated minus green-irradiated samples) of PEC(X) (sample shown in Fig. 5) (a) and of PEC containing fractions from $W$. prolifica (absorption spectra shown in Fig. 4) (b) and $N$. rivulare (c). The "baselines" represent the difference spectra of the ones obtained after a green-orange irradiation sequence, minus that of the original.

adaptochromes and phycomorphochromes, respectively (Bogarad, 1975). The search for photoreversibly photochromic pigments yielded at least four different phycochromes, phycochrome $a, b, c$ and $d$, analogous to the higher plant photoreceptor phytochrome. Among these, phytochrome $b$ gives the largest signals which have ever been detected in 


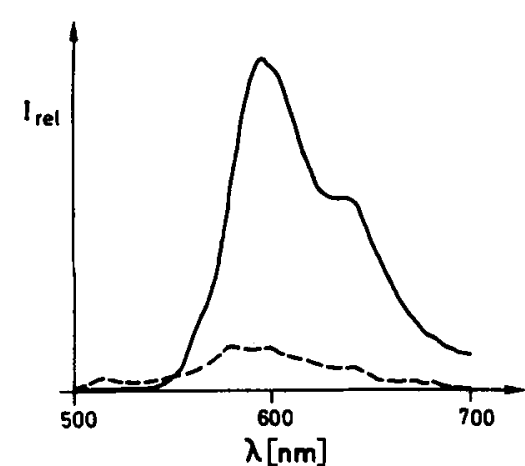

Figure 7. Fluorescence emission spectra of $\operatorname{PEC}(X)$, pre irradiated at $600 \mathrm{~nm}$, excited at $480 \mathrm{~nm}(--)$ and $540 \mathrm{~nm}$ $(\longrightarrow)$. Slitwidths were maintained at $5 \mathrm{~nm}$ for both excitation and emission.

whole cells. This pigment has so far only been found in the PEC containing cyanobacteria, and a relationship or even identity between the PEC $\alpha$-subunit and this phytochrome $b$ has already been suggested (Björn, 1979; Kufer and Björn, 1989; Siebzehnrübl et al., 1989). The $\alpha$-subunit of PEC contains a rare bilin chromophore, phycoviolobilin, which contains a conjugation system intermediate in length between phycocyanobilin and phycoerythrobilin (Bishop et al., 1987). Phycoerythrocyanin is known to dissociate more rapidly than the other cyanobacterial biliproteins (Bryant, 1982) and disaggregation has been shown to be closely related to the amount of reversible photochemistry in PEC from $M$. laminosus (Siebzehnrübl et al., 1989).

The present study was undertaken in order to test if this relationship also holds for PEC from other cyanobacteria. The maximum photochemical signal was found in $\mathrm{PEC}(\mathrm{X})$ fractions from $W$. prolifica, followed by (in this order) PEC monomers, trimers and dissociated PBSomes, and a vanishing signal in the intact PBSomes. These observations regarding the photochemistry of PEC in different states of aggregation from $W$. prolifica are in agreement with those reported for $M$. laminosus by Siebzehnrübl $e t$ al. (1989). However in the latter, a minor signal was found in isolated PBSomes, whereas we could not observe any photochemistry of PEC in intact PBSomes from $W$. prolifica and $N$. rivulare. This substantiates the suggestion that PEC photochemistry is very small or even absent in the PBSome environment. The decrease of photochemistry in higher aggregates has been attributed either to a decreased mobility of the phycoviolobilin chromophore which inhibits the rotation of ring $D$ necessary for isomerization, or to a competition of energy transfer to lower energy phycocyanobilin chromophores with photochemistry (Siebzehnrübl et al., 1989). The pronounced increase of the signal in PEC fractions from $N$. rivulare, when comparing the 1 and $0.1 M$ buffer solutions supports the former mechanism (Table 1). Furthermore, the studies on
PBSomes and biliproteins from $N$. rivulare indicate the presence of PEC in this organism.

The amount of PEC is known to depend on growth conditions; it is, in particular, reduced in high light, but it is also dependent on species (Bryant, 1982). In view of the taxonomic problems in filamentous nitrogen fixing cyanobacteria, PEC has recently been suggested as a marker pigment (Kufer et al., 1990). The results obtained here indicate that reversible photochemistry is a sensitive analytical tool to detect PEC in low amounts and it may thus be helpful in the assignment of species. To test this assay, we have investigated the dissociated PBSomes and isolated phycoerythrins from Anabaena variabilis ARM 310. In agreement with its assignment, no photosignal was observed (data not shown).

A question, which to our knowledge has not yet been addressed, concerns the photophysics of the two forms of the phycoviolobilin chromophore on the $\alpha$-subunit, viz. its fluorescence properties and its involvement in energy transfer. The very pronounced enrichment of either one of them (depending on pre-irradiation) in $\operatorname{PEC}(\mathrm{X})$ from $W$. prolifica, prompted us to study the problem with this pigment. The fluorescence emission properties (Fig. 7) of PEC(X) fractions after $8 \mathrm{~min}$ of $600 \mathrm{~nm}$ illumination indicates that only one form, the longwavelength absorbing one, is fluorescent. The $\mathrm{PEC}(\mathrm{X})$ when excited at $545 \mathrm{~nm}$ emits at $590 \mathrm{~nm}$, characteristic of the native $\alpha$-subunit of PEC. The orange pre-irradiated $\operatorname{PEC}(X)$ also emits at the same wavelength.

However, the intensity of the emission is reduced, and it is further reduced upon excitation at $480 \mathrm{~nm}$. This indicates that the isomer of $\alpha$-subunit absorbing at $510 \mathrm{~nm}$ acts only as an absorber, but has a fast radiationless deactivation channel similar to free bile pigments (Scheer, 1981; Braslavsky et al., 1983). With a photoconversion yield of $4 \%$ (Siebzehnrübl, 1990), this channel dominates the excited state relaxation of chromophores in the $510 \mathrm{~nm}$ form. Accordingly, the involvement of this form in energy transfer in integral PEC or PBSomes may be reduced. It is presently not clear whether the $\operatorname{PEC}(\mathrm{X})$ fraction occurs as a free biliprotein in cyanobacteria or is an isolation artefact. Phycoerythrocyanin is known to dissociate more readily than other biliproteins, which could also explain the large phycochrome $b$ related amplitudes found in vivo

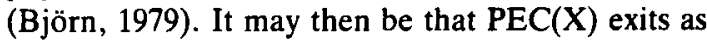
a free biliprotein in vivo.

To summarize our findings, we conclude that in all species of cyanobacteria studied the photochemistry is dependent on the aggregation state of PEC. The negligible photochemical responses of PEC in intact PBSomes suggest that it (or at least the component possessing the long-wavelength absorbing $\alpha$-chromophore) functions only in light harvesting and energy transfer. Our main obser- 
vation, that the $510 \mathrm{~nm}$ absorbing isomer of $\mathrm{PEC}(\mathrm{X})$ does not fluoresce, questions its role in light harvesting for energy transfer purposes and strengthens the possible involvement in light perception when it exists as a free biliprotein.

Finally, it should be noted that the induction of photochemistry of PEC can be used as a sensitive assay for its presence in cyanobacteria, since it is the only biliprotein reported so far to exhibit photochemical responses in the native state.

Acknowledgements-This work was supported by the Deutsche Forschungsemeinschaft, 53 Bonn, Fed. Rep. Germany (SFB 143, Project A1). P.S.M.S. thanks the Council of Scientific and Industrial Research, New Delhi, India for financial support in the form of a Senior Research Fellowship. P.S.M.S. is indebted to Professor H. N. Singh for his helpful discussions.

\section{REFERENCES}

Bishop, J. E., H. Rapoport, A. V. Klotz, C. F. Chan, A N. Glazer, P. Füglistaller and H. Zuber (1987) Chromopeptides from phycoerythrocyanin, structure and linkage of three bilin groups. J. Am. Chem. Soc. 109, 875-881.

Björn, G. S. (1979) Action spectra for in vivo conversions of phycochrome $b$, a reversibly photochromic pigment in a blue-green alga, and its separation from other pigments. Physiol. Plant. 46, 281-286.

Bogarad, L. (1975) Phycobiliproteins and complementary chromatic adaptation. Annu. Rev. Plant. Physiol. 26, 369-401.

Braslavsky, S. E., A. R. Holzwarth and K. Schaffner (1983) Konformationsanalyse, Photophysik und Photochemie der Gallenpigmente; Bilirubin- und Biliverdinmethylester und verwandte lineare Tetrapyrole. Angew. Cherm. 9, 670-689. Angew. Chem. Int. Ed. Engl. 22, $656-674$.

Bryant, D. A. (1982) Phycoerythrocyanin and phycoerythrin properties and occurrence in cyanobacteria. $J$ Gen. Microbiol. 128, 835-844.

Bryant, D. A., A. N. Glazer and F. A. Eiserling (1976) Characterisation and structural properties of major biliproteins of Anabaena sp. Arch. Microbiol. 110, 61-75. Dürring, M., R. Huber, W. Bode, R. Rümbeli and $H$. Zuber (1990) Refined three dimensional structure of phycoerythrocyanin from the cyanobacterium Mastigo- cladus laminosus at $2.7 \AA$. J. Mol. Biol. 211, 633-644.

Gantt, E., C. A. Lipschultz, J. Grabowski and B. K. Zimmermann (1979) Phycobilisomes from blue-green and red algae: isolation criteria and dissociation characteristics. Plant Physiol. 63, 615-620.

Kufer, W. and G. S. Björn (1989) Photochromism of the cyanobacterial light harvesting biliprotein phycoerythrocyanin. Physiol. Plant. 75, 389-394.

Kufer, W., W. Rüdiger and M. Eberlein (1990) Organization, molecular evolution and regulation of the phycoerythrocyanin gene cluster. Abstract of 1st Eur. Workshop on the Molecular Biology of Cyanobacteria, Dourdan, France.

Laemmli, U. K. (1970) Cleavage of structural proteins during the assembly of the head of bacteriophage T4. Nature 227, 680-685.

MacColl, R., K. Csatorday and D. S. Berns (1981) The relationship of the quarternary structure of allophycocyanin to its spectrum. Arch. Biochem. Biophys. 208, 42-48.

MacColl, R. and D. Guard-Friar (1987) Phycobiliproteins. CRC Press, Boca Raton, FL.

Menon, V. K. N., A. Kumar, A. L. Menon and A. K. Varma (1988) A rapid procedure for isolating phycobilisomes from cyanobacteria. Anal. Biochem. 172 $377-381$.

Scheer, H. (1981) Biliproteins. Angew. Chem. 93, 230-250; Angew. Chem. Int. Engl. 20, 241-261.

Scheer, H. (1986) Excitation transfer in phycobiliproteins. In Encyclopedia of Plant Physiology, Vol. 19: Photosynthesis III (Edited by L. A. Staehelin and C. J. Arntzen), pp. 327-337. Springer, Berlin.

Siebzehnrübl, S. (1990). Chromophorzuordnung und reversible Photochemie von C-Phycocyanininen und Phycoerythrocyaninen. Dissertation, Universität München.

Siebzehnrübl, S., R. Fischer, W. Kufer and H. Scheer (1989) Photochemistry of phycobiliproteins: reciprocity of reversible photochemistry and aggregation in phycoerythrocyanin from Mastigocladus laminosus. Photochem. Photobiol. 49, 753-761.

Stanier, R. Y., R. Kunisawa, M. Mandel and G. CohenBazire (1971) Purification and properties of unicellular blue-green algae (order Chroococcales). Bact. Rev. 35, 171-205.

Zuber, H. (1986) Primary structure and function of the light harvesting polypeptides from cyanobacteria, red algae and purple photosynthetic bacteria. In Encyclopedia of Plant Physiology, Vol. 19: Photosynthesis III (Edited by L. A. Staehelin and C. J. Arntzen), pp. 238-251. Springer, Berlin. 